\title{
Tear function and goblet cell density after pterygium excision
}

\begin{abstract}
Purpose To evaluate the short-term effect of pterygium excision on tear function and conjunctival goblet cell density. Methods In all, 70 patients (70 eyes) with primary pterygia who underwent a bare-sclera procedure were enrolled in this study. Tear break-up time, Schirmer, and tear-ferning tests and conjunctival goblet cell density obtained by impression cytology were evaluated before and 1 month after surgery.
\end{abstract}

Results At 1 month postoperation, the tear break-up time (11.49 $\pm 3.76 \mathrm{~s})$ was significantly prolonged when compared with the preoperation tear break-up time $(9.74 \pm 3.43 \mathrm{~s}$, $P=0.002$ ). Before surgery, only $17 \%$ of the patients showed normal crystallization in the tear-ferning test. This proportion was significantly increased to $90 \% 1$ month after surgery $(P<0.001)$. There is no significant difference in the Schirmer test value between pre- and postsurgery $(P>0.05)$. The mean goblet cell density in conjunctival impression specimens before surgery was $41.82 \pm 18.29 / 10$ fields, which was increased significantly 1 month after surgery $(P<0.001)$ with a mean cell density of $50.67 \pm 18.71 / 10$ fields.

Conclusion Tear function in patients with primary pterygium improves after pterygium excision, which indicates that pterygium has a close relationship with dry eye. Eye (2007) 21, 224-228. doi:10.1038/sj.eye.6702186; published online 9 December 2005

Keywords: pterygium; surgery; tear function test; mucus fern test; impression cytology

\section{Introduction}

Pterygium is one of most common ocular surface diseases. The prevalence of this disorder enhanced in some special populations, such as farmers, fishermen, and people living in dry,

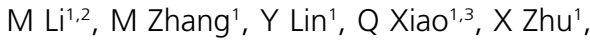
S Song', J Lin', J Chen' and Z Liu' sunny clients, etc. ${ }^{1}$ Although the aetiology of pterygium is still not fully understood, prolonged exposure to ultraviolet $B$ radiation is thought to promote its development. ${ }^{2,3}$ Other factors such as inherited susceptibility, ${ }^{4}$ chronic inflammation, ${ }^{5}$ heat, ${ }^{6}$ and still others may also play a role in the formation of pterygium. Kadayifcilar $^{7}$ and Ishioka ${ }^{8}$ noted inadequate tear film stability in pterygia patients and suggested that abnormal tear function may be yet another risk factor related to pterygium development. However, several other studies have shown that tear function is normal in pterygia. ${ }^{9-11}$ Thus, there is an unresolved issue with regard to whether the abnormal tear function is directly associated with pterygium. If they are related, another question arises: does the change of the tear function cause pterygium or vice versa?

Surgical removal of pterygium is a commonly accepted treatment for this disorder. Research data on the effects of pterygium excision on refraction and ocular surface epithelium are widely available. ${ }^{12,13}$ However, there is no report on the effect of pterygium excision on tear function. The present study is conducted to determine whether surgical removal of pterygium can cause any changes in tear function and conjunctival goblet cell density in patients with primary pterygium.

\section{Materials and methods}

This study was conducted according to the tenets of the Declaration of Helsinki. Informed consent was obtained from each subject after a full explanation of the nature and possible consequences of the procedures used in the study.

\section{Patients}

In this prospective nonrandomized (selfcontrolled) comparative trial, 70 eyes of 70 
patients with pterygia were enrolled from the Zhongshan Ophthalmic Center outpatient service between November 2001 and March 2002. Ophthalmic examinations, including slit-lamp biomicroscopy and tear film evaluation, were performed on all enrolled subjects. Subjects with previous ocular surgery, contact lens wear, pemphigoid, Sjögren's syndrome, or any other type of conjunctivitis or keratitis were excluded from the study.

All 70 cases were primary pterygia patients, consisting of 36 men (36 eyes) and 34 women ( 34 eyes). The mean age was $54 \pm 15$ years (mean age $\pm S D$ ) (range, 24-82), with 35 right eyes and 35 left eyes. All pterygia were in the nasal part of the lid fissure. Pterygium excision with a 'baresclera' procedure was carried out for every patient in this study. Patients were followed up for at least 1 month.

\section{Surgical procedures}

Pterygium excision was performed by the same surgeon (Dr Li M). The bare-sclera technique was applied on each patient as described previously. ${ }^{14}$ Briefly, topical anaesthesia was performed with $0.5 \%$ Alcaine (Alcon, Texas, USA). After applying an eyelid speculum, 2\% lidocaine hydrochloride was injected under the pterygium to elevate it from the cornea. With the help of toothed forceps, the body of pterygium was grasped and the excision was performed with a blade, starting from the corneal side. A complete resection of the mass was performed, and the dissociated edges of conjunctiva were interruptedly sutured, leaving a bare-sclera area of $4 \times 4 \mathrm{~mm}^{2}$. At the completion of surgery, $0.3 \%$ tobramycin and $0.1 \%$ dexamethasone ointment were applied topically, followed by patching.

All eyes were treated with $0.3 \%$ tobramycin and $0.05 \%$ dexamethasone eye drops four times a day, and $0.3 \%$ tobramycin ointment before sleeping at night. The ointment was stopped 1 week after surgery, and the eye drops were discontinued 7-10 days postoperation. Sutures were removed 5 days after surgery. Artificial tears were not used before surgery or during follow-up.

\section{Evaluation of tear film function}

All examinations were performed in the morning and all variables of each patient were evaluated at one office visit in the same dark room 1 day before surgery and 1 month postoperatively. Tear film break-up time measurement with fluorescein and the Schirmer test without topical anaesthesia were performed. Tear film break-up time was recorded as the average of three successive measurements. Eyes with dry spots consistently localized in the same corneal area were excluded from the study. The Schirmer test result was expressed as the wet length of the strip measured after $5 \mathrm{~min}$. After a break of $30 \mathrm{~min}, 2-3 \mu \mathrm{l}$ of nonstimulated tear was collected with a capillary pipette from the lower fornix without anaesthesia to evaluate the ferning pattern. Care was taken not to grasp the conjunctival surface. The crystallization patterns were classified into four groups (Figure 1a-d): ${ }^{7}$ Type I: Uniform and closely branching arborization. Type II: Single ferns are smaller and less branching is present. Type III: Small ferns with almost no branches, many empty spaces. Type IV: Ferning is absent and clusters of crystal may be present.

\section{Conjunctival impression cytology}

Conjunctival impression was conducted after tear film function evaluation. Specimens were collected one day before and one month after pterygium excision. The techniques for impression cytology specimen collection, preparation, and examination have been described previously. ${ }^{13}$ In brief, the nitrocellulose filter strips $(3 \times 3 \mathrm{~mm})$ with a pore size of $0.22 \mu \mathrm{m}$ were soaked in distilled water for 3-4h and dried at room temperature before use. After administration of topical anaesthesia with $0.5 \%$ Alcaine, a strip was applied to the surface of the superior bulbar conjunctiva, $3 \mathrm{~mm}$ away from the limbus, and then pressed gently by a glass rod. Excess tears in the inferior conjunctival fornix were gently absorbed with cotton wipes before the nitrocellulose strip was peeled off. The specimens were immediately fixed with $95 \%$ ethanol and stained with periodic acid-SchiffGill's modified Papanicolaou staining. Under the microscope, goblet cells were stained by PAS with red colour. The mean goblet cell density for each specimen was determined by averaging the total number of goblet cells in 10 consecutive visual fields of high magnification $(\times 400)$. Among the 70 patients, 15 patients were excluded from the analysis because of the failure to obtain any cells. Therefore, blotting cytology data of 55 subjects were analysed. All conjunctival impression cytology specimens were obtained and evaluated in a masked fashion by the same technician (Lin J).

\section{Statistics}

The data were processed using SPSS 10.0 software (SPSS Inc., Chicago, IL). The Paired-Samples $t$-test or $\chi^{2}$ test was used to compare the measurements pre- and postoperatively. A $P$-value $<0.05$ was considered statistically significant.

\section{Results}

\section{Clinical evaluation}

During the follow-up time, no serious ocular complications such as infection or scleral necrosis were 

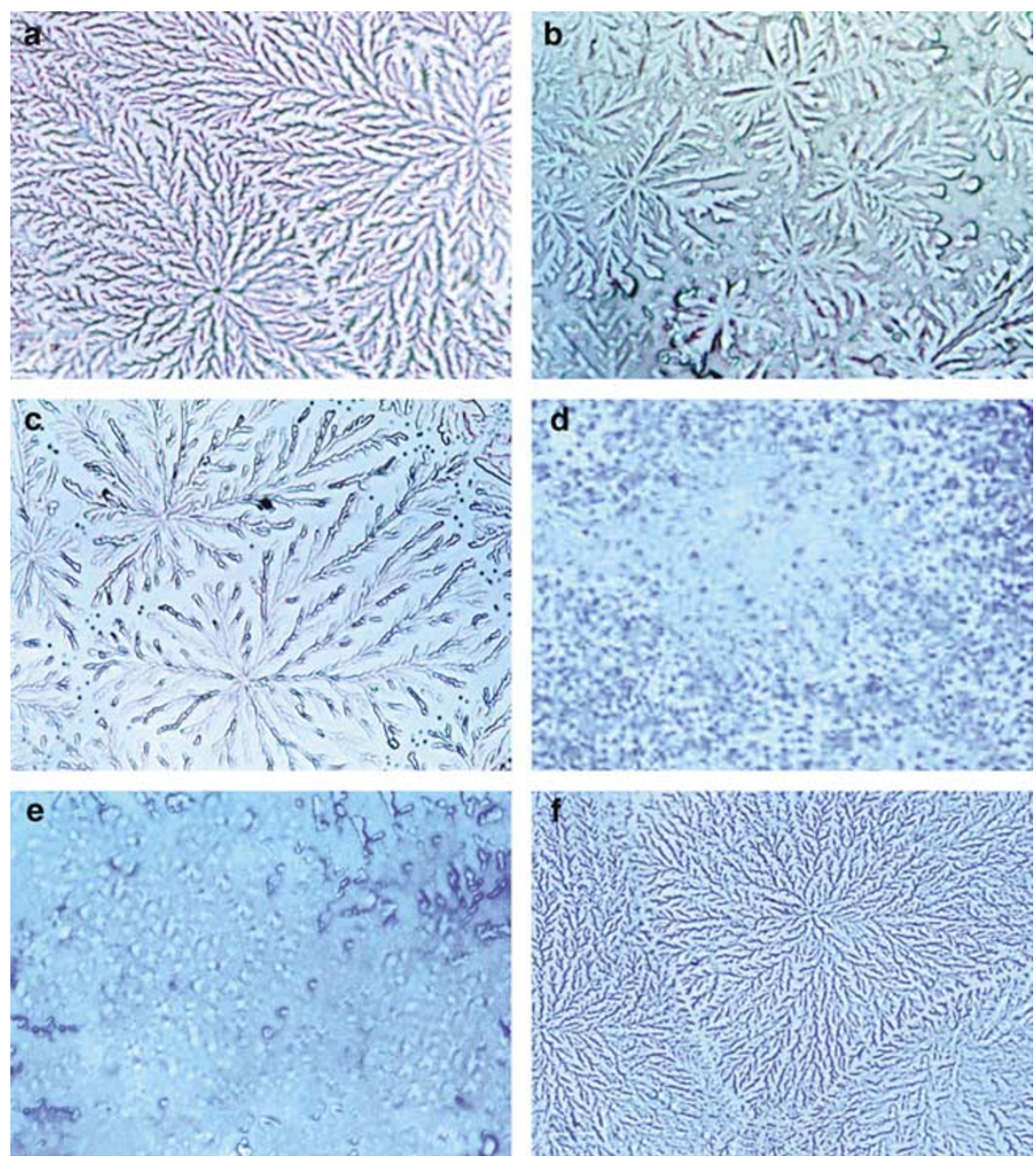

Figure 1 In figure $(a-d)$ shows the four types of crystallization patterns of tear ferning. The crystallization patterns were classified into four groups: (a) Type I. Uniform and closely branching arborization. (b) Type II. Single ferns are smaller and less branching is present. (c) Type III. Small ferns with almost no branch; many empty spaces. (d) Type IV. Ferning is absent and clusters of crystal may be present. (e) and (f) show tear ferning patterns of a 56-year-old female patient before and 1 month after pterygium excision. (e) Type IV of tear ferning before pterygium excision. (f) Tear ferning recovered to type I, 1 month after surgery in the same patient as in (e). Magnification, $\times 400$.

observed in any eye that received surgery. Corneal epithelial defects healed within 4-7 days after surgery.

\section{Tear film function}

Before surgery, the average tear break-up time was $9.74 \pm 3.43 \mathrm{~s}$. This was significantly prolonged to $11.49 \pm 3.76 \mathrm{~s} 1$ month postoperatively $(t=3.28, P=0.002$, Paired-Sample $t$-test). The distribution of tear-ferning types before and after surgery is shown in Table 1. Before surgery, only 12 of the 70 patients (17\%) had normal crystallization (Type I and II) of tear ferning, while $90 \%$ (63/70) patients showed normal tear ferning 1 month after surgery. The difference is statistically significant $\left(\chi^{2}=74.695, P<0.001, \chi^{2}\right.$ test $)$. Figure 1e and $\mathrm{f}$ demonstrate the recovery of tear crystallization in a 56-year-old female patient. There was no statistically significant difference for the Schirmer test results before and after surgery $(P>0.05)$.

\section{Impression cytology}

The mean goblet cell density in conjunctival impression specimens was $41.82 \pm 18.29 / 10$ fields before surgery. It was significantly increased to $50.67 \pm 18.71 / 10$ fields 1 month after surgery $(t=6.37, P<0.001$, Paired-Samples $t$-test ). Figure 2 shows the increase of goblet cell density after pterygium excision in a 44-year-old female patient.

\section{Discussion}

The relationship between pterygia and tear film function has proved difficult to define. Decreased tear break-up time, abnormal tear-ferning types and/or insufficient tear secretion in some pterygia patients have been noted 
Table 1 Distribution of tear-ferning types before and after surgery

\begin{tabular}{lcccccc}
\hline & \multicolumn{5}{c}{ Tear-ferning type (case number) } \\
\cline { 2 - 7 } & $I$ & $I I$ & Total & III & IV & Total $^{\text {b }}$ \\
\hline 1 day before surgery & $4(6 \%)$ & $8(11 \%)$ & $12(17 \%)$ & $26(37 \%)$ & $32(46 \%)$ & $58(83 \%)$ \\
1 month after surgery & $28(40 \%)$ & $35(50 \%)$ & $63(90 \%)^{\text {c }}$ & $3(4 \%)$ & $4(6 \%)$ & $7(10 \%)$ \\
\hline
\end{tabular}

aTotal: Type I plus type II.

${ }^{\text {b}}$ Total: Type III plus type IV.

${ }^{c} \chi^{2}=74.695, P<0.001$.
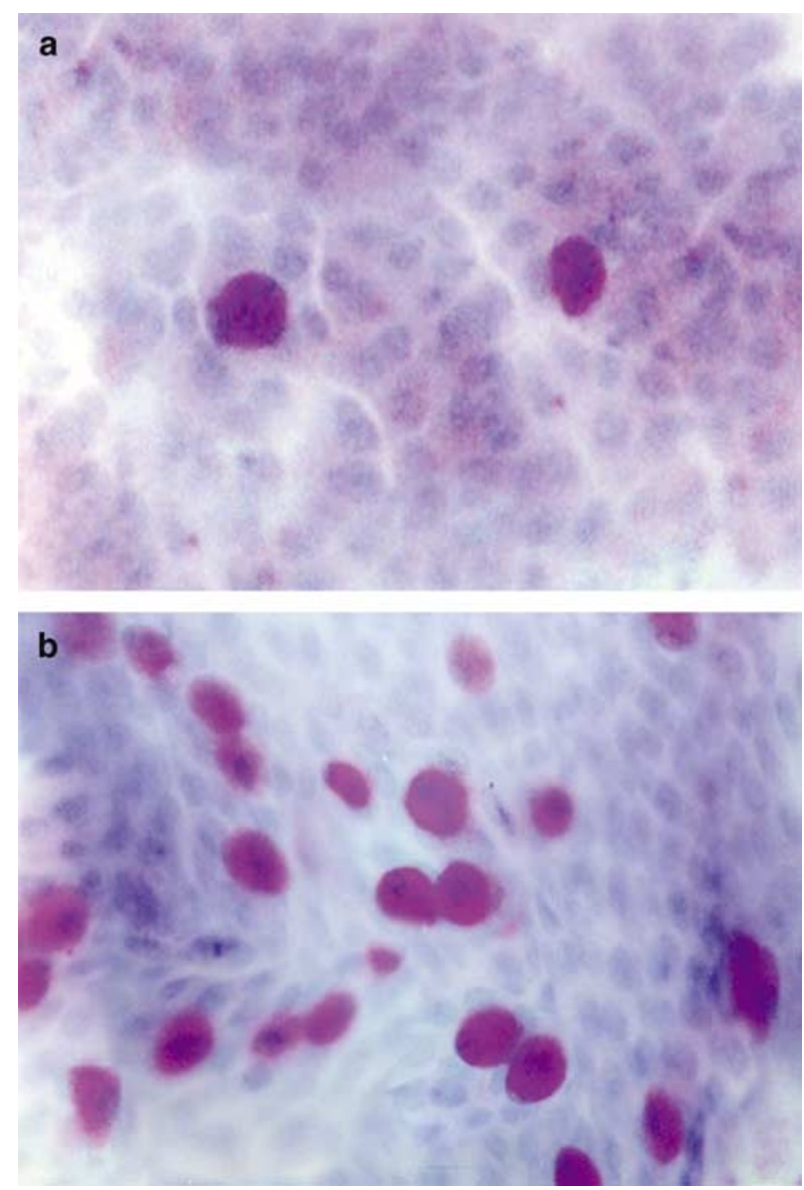

Figure 2 Conjunctival impression cytology of a 44-year-old female pterygium patient before and 1 month after surgery. Conjunctival goblet cells were stained by PAS with purple red cytoplasm. Less conjunctival goblet cells were present before surgery (a), while the goblet cell density increased significantly 1 month after pterygium excision (b). Magnification, $\times 400$.

by some but not all investigators. ${ }^{7,8,15-17}$ Since the preocular tear film is the first line of defence against environmental injury, including ultraviolet exposure (which is believed to be the key factor of pterygium development), many authors hypothesize that an abnormal tear function is a risk factor for diseases resulting from ultraviolet exposure, including pterygium. In contrast, other authors suggest the reverse sequence: pathological conjunctival, corneal, or eyelid changes in pterygia lead to disturbed tear film function. ${ }^{18}$ Based on the possibility of co-occurrence of dry eye and pterygia, artificial tear therapy was introduced in an effort to prevent the development of pterygium. $^{19}$

However, the result is unknown: no report on the effect of artificial tears on the formation or progression of pterygium is available.

In still other studies, no differences of tear function between pterygia affected and healthy eyes were found at all. ${ }^{9-11,20}$ These authors inferred that dryness of the eyes cannot be regarded as the cause of pterygium. Recently, a two-fold increased risk of dry eye symptoms was noted in pterygia patients. The author hypothesized that the ultraviolet light quantities and dusty polluted environment of outdoor work, which were implicated in pterygium formation, may also possibly become the risk factors to dry eye formation in pterygia patients. ${ }^{21}$

In the present study, alterations of tear function were compared before and after pterygium excision. To our knowledge, this is the first report on the effects of pterygium surgery on tear film function. Tear function was found to be partially restored: tear break-up time, tear ferning, and conjunctival goblet cell density all improved. Thus, the results of the present study suggest that once formed, pterygium itself leads to abnormal tear film function.

Besides the aforementioned environmental factors suggested by Lee, ${ }^{21}$ several factors related to pterygium may also contribute to the abnormality of tear function, including abnormal tear dynamics and composition. For tear dynamics, a recent study has shown that there is a pooling of tears at the pterygium apex as evidenced by the significantly increased total corneal refractive power after the tears were dried. ${ }^{22}$ The pooling of tears, presumably caused by the protrusion of pterygial lesion, can disturb the distribution of tear film on the ocular surface. It is still unclear whether the tear volume plays a role in the abnormal tear function in pterygia patients. Decreased tear production has been reported in some studies, ${ }^{8,16,17}$ while the unchanged tear volume was reported in other studies. ${ }^{7,10,11,15,20}$ In our study, no difference was noted in Schirmer test value before and after pterygium excision. With respect to the components 
of tear film, both Kadayifcilar ${ }^{7}$ and Marzeta ${ }^{15}$ have shown a high frequency of abnormal tear-ferning type. We also found that the distribution of tear-ferning types changed remarkably after surgical removal of the pterygial lesion. Few patients had normal tear ferning before surgery, while most recovered normal tear ferning after excision. The reduction of tear ferning in patients with pterygium is attributed to the alteration of chemical compositions of the tears such as the deficiency of mucus. ${ }^{7,15}$ Thus, the reduced tear ferning in pterygia patients and its recovery after pterygium excision clearly indicate that an alternation of tear composition contributes to the dry eye condition. A recent study suggests that it is the balance of macromolecules and inorganic salts rather than the level of single species that gives rise to the tear ferning. ${ }^{23}$ Nevertheless, the recovered tear ferning in pterygia patients suggests a change of tear composition caused by pterygium excision.

Lastly, ocular surface changes in pterygium and the manifestation of healing ocular surface obtained by impression cytology after excision have been well documented. ${ }^{13,24}$ In Chan's study, mucinous hyperplasia signifying increased goblet cell density was observed throughout the bulbar conjunctiva, most occurring just over the pterygium (88\%) and the least in the superior conjunctiva $(2 \%) .{ }^{24}$ The effect of pterygium excision on goblet cell density has not been reported. In our study, impression specimens were taken from the superior bulbar conjunctiva, where the epithelial cells are considered to be the least affected by pterygia. The mean goblet cell density was found significantly increased one month after excision, which may result in an increased secretion of mucin (mainly MUC5AC) in tear film.

Our results, taken together with previous data, suggest that pterygium has a close relationship with dry eye, although it still remains unclear which disorder is causative. It is likely that the two disorders may affect each other's development. Further investigation is needed to clarify these questions.

\section{Acknowledgements}

Grant Information: This study was supported by grants from National Natural Science Foundation of China (2003, 30371514) and National Science Fund for Distinguished Young Scholars of China (2002).

\section{References}

1 Detels R, Dhir SP. Pterygium: a geographical study. Arch Ophthalmol 1967; 78: 485-491.
2 Mackenzie FD, Hirst LW, Battistutta D, Green A. Risk analysis in the development of pterygia. Ophthalmology 1992; 99: 1056-1061.

3 Saw SM, Banerjee K, Tan D. Risk factors for the development of pterygium in Singapore: a hospital-based case-control study. Acta Ophthalmol Scand 2000; 78: 216-220.

4 Islam SI, Wagoner MD. Pterygium in young members of one family. Cornea 2001; 20: 708-710.

5 Hill JC, Maske R. Pathogenesis of pterygium. Eye 1989; 3(Part 2): 218-226.

6 Young R. The family of sunlight-related eye diseases. Optom Vis Sci 1994; 71: 125-144.

7 Kadayifcilar SC, Orhan M, Irkec M. Tear functions in patients with pterygium. Acta Ophthalmol Scand 1998; 76: 176-179.

8 Ishioka M, Shimmura S, Yagi Y, Tsubota K. Pterygium and dry eye. Ophthalmologica 2001; 215: 209-211.

9 Taylor HR. Studies on the tear film in climatic droplet keratopathy and pterygium. Arch Ophthalmol 1980; 98: 86-88.

10 Ergin A, Bozdogan O. Study on tear function abnormality in pterygium. Ophthalmologica 2001; 215: 204-208.

11 Biedner B, Biger Y, Rothkoff L, Sachs U. Pterygium and basic tear secretion. Ann Ophthalmol 1979; 11: 1235-1236.

12 Fong KS, Balakrishnan V, Chee SP, Tan DT. Refractive change following pterygium surgery. Clao J 1998; 24: 115-117.

13 Tseng SH, Chen YT, Cheng HC, Huang FC, Lee SC, Chen FK. Impression cytology study of conjunctival epithelial phenotypes on the healing ocular surface after pterygium excision. Cornea 2001; 20: 244-250.

14 Panda A, Das GK, Tuli SW, Kumar A. Randomized trial of intraoperative mitomycin C in surgery for pterygium. Am J Ophthalmol 1998; 125: 59-63.

15 Marzeta M, Toczolowski J. Study of mucin layer of tear film in patients with pterygium. Klin Oczna 2003; 105: 60-62.

16 Chaidaroon W, Pongmoragot N. Basic tear secretion measurement in pterygium. J Med Assoc Thai 2003; 86: 348-352.

17 Rajiv, Mithal S, Sood AK. Pterygium and dry eye - a clinical correlation. Indian J Ophthalmol 1991; 39: 15-16.

18 Brewitt H, Sistani F. Dry eye disease: the scale of the problem. Surv Ophthalmol 2001; 45: S199-S202.

19 Joros PA, Deluise VP. Pingueculae and pterygia. Surv Opthalmol 1988; 33: 41-49.

20 Goldberg L, David R. Pterygium and its relationship to the dry eye in the Bantu. Br J Ophthalmol 1976; 60: $720-721$.

21 Lee AJ, Lee J, Saw SM, Gazzard G, Koh D, Widjaja D et al. Prevalence and risk factors associated with dry eye symptoms: a population based study in Indonesia. $\mathrm{Br} \mathrm{J}$ Ophthalmol 2002; 86: 1347-1351.

22 Yasar T, Ozdemir M, Cinal A, Demirok A, Ilhan B, Durmus AC. Effects of fibrovascular traction and pooling of tears on corneal topographic changes induced by pterygium. Eye 2003; 17: 492-496.

23 Pearce EI, Tomlinson A. Spatial location studies on the chemical composition of human tear ferns. Ophthalmic Physiol Opt 2000; 20: 306-313.

24 Chan CM, Liu YP, Tan DT. Ocular surface changes in pterygium. Cornea 2002; 21: 38-42. 